\title{
The Role of Peripheral Blood Inflammatory Markers in
}

\section{the Staging of Breast Cancer}

\author{
Fevzi Coşkun Sökmen ${ }^{1^{\star}}$, Cengiz Karacin ${ }^{2}$ \\ ${ }^{1}$ University of Health Science, Dr Abdurrabman Yurtaslan Oncology Training and Research Hospital, Department of \\ Internal Medicine \\ ${ }^{2}$ University of Health Science, Dr Abdurrahman Yurtaslan Oncology Training and Research Hospital, Department of \\ Medical Oncology
}

\begin{abstract}
This study aims to evaluate the importance of neutrophil-lymphocyte ratio (NLR), lymphocyte-monocyte ratio (LMR), platelet-lymphocyte ratio (PLR), and systemic immune-inflammatory index (SII) in the staging of breast cancer.

One-hundred patients with breast cancer between January 2005 and December 2019 were reviewed retrospectively. Clinical and demographic data of patients were reviewed from the hospital records. Age, gender, pre-treatment complete blood count results and stages were analyzed from patient files.

The median NLR, PLR, and SII values of the T3 stage were higher than the T1 and T2 stages. While there was no difference between the groups according to the $\mathrm{N}$ stage, a difference was found between the peripheral blood inflammatory markers of Stage I, II, and III groups. The median NLR, PLR, and SII values of stage III were higher than the stage II and III.

As a result, NLR, PLR, LMR, and SII may be valuable in breast cancer staging.
\end{abstract}

Key Words: Breast Cancer, Neutrophil-to-lymphocyte ratio, Lymphocyte-to-monocyte ratio, Platelet-to-lymphocyte ratio, Systemic immune-inflammatory index

\section{Introduction}

Breast cancer (BC), the most common cancer in women, leads to cancer-related deaths between the ages of 40 and 59 (1). It is thought that inflammation caused by cancer is a prognostic factor, and studies on this subject have increased in recent years. The leukocytes and tumorassociated macrophages in the neoplastic tissue microenvironment, play a crucial role. Transcription factors, signal converter, and transcription-3 activator, cytokines, and their chemokines lead to tumor angiogenesis, tumor cell survival and proliferation (2).

Studies linking neutrophil-lymphocyte ratio (NLR), lymphocyte-monocyte ratio (LMR), and platelet-lymphocyte ratio (PLR) to cancers are increasing (3-5). Although there are studies about the prognostic importance of these values in breast cancer, very few of them investigated inflammatory markers in staging $(6,7)$. To the best of our knowledge, there is no study examining the role of NLR, PLR, LMR, and SII in BC staging. This study aims to investigate the importance of
NLR, LMR, PLR, and SII in the staging of breast cancer.

\section{Material and Methods}

One-hundred patients with breast cancer between January 2005 and December 2019 were reviewed retrospectively. Patients with previously untreated breast cancer who had medical and follow-up records were included in the study. Metastatic disease, high CRP levels, diabetes mellitus, infectious diseases, rheumatologic diseases, inflammatory diseases, hematological disease, usage of non-steroid anti-inflammatory drugs, and missing medical records were the exclusion criteria of the study. Clinical and demographic data of patients were reviewed from the hospital records. Age, gender, pre-treatment, complete blood count results, and tumor stages were analyzed from patient files. NLR and PLR were calculated as the absolute count of neutrophils and platelets, divided by the absolute lymphocyte count. LMR was calculated as the absolute count of lymphocyte divided by the absolute monocyte 
Table 1. Demographic characteristics of the patients

\begin{tabular}{lc}
\hline Age, median (range) & $55(29-79)$ \\
Histopathology, n (\%) & \\
Invasive ductal carcinoma & $87(87)$ \\
Invasive lobular carcinoma & $13(13)$ \\
T stage, n (\%) & \\
T1 & $27(27)$ \\
T2 & $58(58)$ \\
T3 & $15(15)$ \\
N stage, n $\%)$ & \\
N0 & $48(48)$ \\
N1 & $35(35)$ \\
N2 & $14(14)$ \\
N3 & $3(3)$ \\
Stage & \\
I & $17(17)$ \\
II & $65(65)$ \\
III & $18(18)$ \\
\hline
\end{tabular}

Table 2. Comparison of NLR, LMR, PLR, and SII levels of patients according to T stage

\begin{tabular}{lcccc}
\hline & T1 & T2 & T3 & P \\
& $\mathrm{n}: 27$ & $\mathrm{n}: 58$ & $\mathrm{n}: 15$ & \\
\hline NLR & $1.82(1.33-2.50)$ & $1.86(1.55-2.34)$ & $3.15(2.33-4.41)$ & 0.001 \\
LMR & $5.10(4.14-7.21)$ & $5.39(4.44-6.63)$ & $4.19(3.16-5.25)$ & 0.087 \\
PLR & $116(92.7-142.2)$ & $126(97.7-167.0)$ & $174(121.6-207.5)$ & 0.023 \\
SII & $546(339.5-867.5)$ & $530(425.1-676.0)$ & $938(607.8-1172.5)$ & 0.014 \\
\hline
\end{tabular}

NLR, Neutrophil-lymphocyte ratio; LMR, Lymphocyte-monocyte ratio; PLR, Platelet-lymphocyte ratio; SII, systemic immune-inflammation index

count. SII was calculated by multiplying the NLR by the number of platelets.

Statistical Analysis: Statistical analyses performed via IBM SSPS Statistics for Windows v. 20.0 software (IBM Corp., Armonk, NY). In descriptive statistical analyses, frequencies (percentages) was used for categorical variables; median (interquartile range) was used for nonparametric variables. Kruskal-Wallis test was used for comparisons of continuous variables. When a statistical difference was detected in the KruskalWallis test, the groups were evaluated in pairs with the Mann-Whitney-u test. Thus, it was determined which groups made the difference. A $p$ value of $<0.05$ was considered statistically significant.

\section{Results}

The median age of patients with breast cancer $(87$ IDC, 13 ILC) included in the study was 55 years (range: 29-79). The demographic characteristics and TNM staging of the patients are shown in Table 1.

The patients were divided into three groups according to the $\mathrm{T}$ stage as $\mathrm{T} 1, \mathrm{~T} 2$, and $\mathrm{T} 3$. When the patients were evaluated according to the $\mathrm{T}$ stage, statistically significant differences were found between the groups (Table 2). The median NLR levels of those with tumor stage T3 were higher than those with T1 $(\mathrm{p}=0.004)$ and $\mathrm{T} 2$ $(p=0.002)$. The median PLR value of patients with stage T3 was higher than patients with stage T1 ( $p$ $=0.019)$. The median SII value of $\mathrm{T} 3$ stage patients was significantly higher than the T2 stage patients $(\mathrm{p}=0.011)$.

While there was no difference between the groups according to the $\mathrm{N}$ stage, a difference was found between the peripheral blood inflammatory markers of Stage I, II, and III groups (Table 3 and 4).

The median NLR, PLR, and SII values of stage III were higher than the stage II and I (Table 4). 
Table 3. Comparison of NLR, LMR, PLR, and SII levels of patients according to the N stage

\begin{tabular}{lcccc}
\hline & $\mathrm{N} 0$ & $\mathrm{~N} 1$ & $\mathrm{~N} 2-3$ & $\mathrm{p}$ \\
$\mathrm{n}: 48$ & $\mathrm{n}: 35$ & $2.69(1.91-3.16)$ & 0.067 \\
\hline NLR & $1.88(1.51-2.37)$ & $1.80(1.42-2.54)$ & $4.33(3.96-6.63)$ & 0.238 \\
LMR & $5.63(4.43-7.02)$ & $4.92(4.18-5.95)$ & $164(102.1-202.7)$ & 0.100 \\
PLR & $117(95.3-155.5)$ & $130(106.9-168.5)$ & $792(501.8-1094.0)$ & 0.116 \\
SII & $541(339.4-697.4)$ & $590(368.2-760.6)$ & 790 \\
\hline
\end{tabular}

NLR, Neutrophil-lymphocyte ratio; LMR, Lymphocyte-monocyte ratio; PLR, Platelet-lymphocyte ratio; SII, systemic immune-inflammation index

Table 4. Comparison of NLR, LMR, PLR, and SII levels of patients according to disease stage

\begin{tabular}{lcccc} 
& I & II & III & p \\
& $\mathrm{n}: 17$ & $\mathrm{n}: 65$ & $\mathrm{n}: 18$ & \\
\hline NLR & $1.38(1.27-2.05)$ & $1.89(1.56-2.49)$ & $2.69(1.91-3.00)$ & 0.016 \\
LMR & $6.72(5.68-7.81)$ & $5.06(4.16-6.02)$ & $4.57(3.96-6.63)$ & 0.021 \\
PLR & $98(82.5-134.9)$ & $132(104.6-170.6)$ & $157(102.0-207.6)$ & 0.046 \\
SII & $435(305.4-640.7)$ & $555(426.0-743.9)$ & $749(501.8-1094.0)$ & 0.068 \\
\hline
\end{tabular}

NLR, Neutrophil-lymphocyte ratio; LMR, Lymphocyte-monocyte ratio; PLR, Platelet-lymphocyte ratio; SII, systemic immune-inflammation index

Stage III group's median NLR value was higher than Stage I $(\mathrm{p}=0.012)$. The median LMR value of the Stage I group was higher than Stage II and III groups (p-value was 0.034 and 0.036 , respectively).

\section{Discussion}

In this study, we evaluated the role of peripheral inflammatory markers in breast cancer staging. The study findings showed that NLR, PLR, LMR, and SII are markers that can aid in breast cancer staging. It has been demonstrated that high NLR, PLR, SII, and low LMR may be associated with the advanced TNM stage.

$\mathrm{BC}$ is a highly heterogeneous disease that offers a wide variety of clinicopathological features. Patients with stage I-III disease classified by the International Association for Cancer Controls (UICC) require mastectomy/lumpectomy +/adjuvant chemotherapy. There is a $10 \%$ to $40 \%$ chance of recurrence after definitive treatment, depending on the underlying molecular subtype of the tumor and the TNM stage (8). Although there are many studies in the literature evaluating the relationship between inflammatory markers and cancer prognosis, studies evaluating these markers' relationship with tumor stage are very rare (6). Elyasinia et al. showed a correlation between NLR and $\mathrm{T}$ stage in patients with BC (6). In our study, the median NLR, PLR, and SII values of the T3 stage were higher than the T1 and T2 stages.
In a study showing that NLR may be associated with lymph node metastasis in breast cancer, it has been shown that high NLR can predict lymph node metastasis in patients with axillary lymph node dissection, although sentinel lymph node biopsy is negative (9). Our study found that the median NLR of the N2-3 group was in an increasing trend compared to the $\mathrm{N} 0$ and N1 groups. We thought that the low number of patients in the N2-3 group might have affected this result.

Neutrophils suppress the lymphocytes', NK cells', and activated T cells' cytolytic activity to inhibit the immune system. Also, a low lymphocyte count has negative effect in patients with cancer. Host cell-mediated immunity is expected to have significant effects in destroying micrometastases and remaining tumor cells. Tumor infiltration by lymphocytes has been reported to demonstrate an effective antitumor cellular immune response and increased lymphocyte infiltration associated with a better prognosis $(10,11)$.

Recent studies have reported that tumor size, pathologic tumor, node, metastasis (TNM) staging is significantly associated with the prognosis of patients with breast cancer (12). However, most of these factors are often obtained under the requirement of core biopsies and with the aid of molecular technique, which is invasive and costly. Based on this idea, we found that cheaper and more easily measurable NLR, PLR, and LMR values can guide us in staging. 
The main limitations of this study are that it is a retrospective study and a single-center experience. Also, the number of patients in this study is low.

This study's main finding is that NLR, PLR, LMR, and SII values, which can be obtained with a simple complete blood count, can guide the staging of BC. NLR, PLR, and LMR may be valuable in breast cancer staging, but more studies are needed in this area.

\section{References}

1. Siegel R, Ward E, Brawley O, Jemal A. Cancer statistics, 2011: the impact of eliminating socioeconomic and racial disparities on premature cancer deaths. CA Cancer J Clin 2011; 61: 212236.

2. Mantovani A, Allavena P, Sica A, Balkwill F. Cancer-related inflammation. Nature 2008; 454: 436-444.

3. Dirican A, Kucukzeybek BB, Alacacioglu A, et al. Do the derived neutrophil to lymphocyte ratio and the neutrophil to lymphocyte ratio predict prognosis in breast cancer? Int J Clin Oncol 2015; 20: 70-81.

4. Azab B, Bhatt VR, Phookan J, et al. Usefulness of the neutrophil-to-lymphocyte ratio in predicting short-and long-term mortality in breast cancer patients. Ann Surg Oncol 2012; 19: 217-224.

5. Ethier J-L, Desautels D, Templeton A, Shah PS, Amir E. Prognostic role of neutrophil-tolymphocyte ratio in breast cancer: a systematic review and meta-analysis. Breast Cancer Res 2017; 19: 1-13.

6. Elyasinia F, Keramati MR, Ahmadi F, et al. Neutrophil-Lymphocyte Ratio in Different Stages of Breast Cancer. Acta Med Iran 2017; 55: 228232.

7. Templeton AJ, McNamara MG, Šeruga B, et al. Prognostic role of neutrophil-to-lymphocyte ratio in solid tumors: a systematic review and metaanalysis. JNCI: Journal of the National Cancer Institute 2014; 106

8. Group EBCTC. Comparisons between different polychemotherapy regimens for early breast cancer: meta-analyses of long-term outcome among 100000 women in 123 randomised trials. Lancet 2012; 379: 432-444.

9. Ibrahim A, Serkan YF, Tuba A, Erol B, Lütfi P. Can neutrophil to lymphocyte ratio be a predictor tool for the non-sentinel lymph node metastasis in breast cancer. Chirurgia (Bucur) 2019; 114: 8388.

10. Fogar P, Sperti C, Basso D, et al. Decreased total lymphocyte counts in pancreatic cancer: an index of adverse outcome. Pancreas 2006; 32: 22-28.

11. Yamanaka T, Matsumoto S, Teramukai S, Ishiwata R, Nagai Y, Fukushima M. The baseline ratio of neutrophils to lymphocytes is associated with patient prognosis in advanced gastric cancer. Oncology 2007; 73: 215-220.

12. Lyman GH, Temin S, Edge SB, et al. Sentinel lymph node biopsy for patients with early-stage breast cancer: American Society of Clinical Oncology clinical practice guideline update. J Clin Oncol 2014; 32: 1365-1383. 